\title{
Comportement dynamique des labyrinthes dans les machines hydrauliques
}

\author{
Dynamic behaviour of ring seals \\ in hydraulic turbomachinary
}

\author{
A. Verry \\ EDF-DER \\ 6, quai Watier \\ 78400 Châtou
}

P. Guiton

Retraité

Bergeron S.A.

L'influence des labyrinthes et bagues d'étanchéité sur le comportement dynamique des rotors de turbomachines est ressentie depuis longtemps par les constructeurs, les expérimentateurs et les utilisateurs.

Après une description des principaux phénomènes physiques régissant le comportement dynamique des labyrinthes, on présente une analyse critique des principaux modèles qui ont été développés (modèle de Lomakin, analyse linéaire de Black, résolution numérique des équations de Hirs développée par DW. Childs). Ensuite, sont étudiées quelques configurations qui peuvent conduire à des instabilités.

En conclusion, on met l'accent sur les aspects qui devront être approfondis pour répondre aux problèmes industriels actuels.

The influence of ring seals on rotor dynamic of hydraulic turbomachinery has been felt for a long time by manufacturers, experimentators and users.

After a description of the main phenomena which govern dynamic behaviour of ring seals, one presents the main theoretical models (Lomakin model, Black linear analysis, numerical resolution of Hirs equations developped by Childs). Then some unstable configurations are studied.

In conclusion, one emphasizes the points which are to be deepened to cope with today industrial problems.

1. - Introduction

2. - Différents types de labyrinthes

3. - Raideur hydrostatique sans rotation (effet Lomakin)

3.1. Écoulement entre deux cylindres coaxiaux (jeu radial constant)

3.2. Raideur hydrostatique

3.3. Limite de validité et développements complémentaires

4. - Amortissement et inertie sans rotation

5. - Influence de la rotation avec écoulement de Couette

5.1. Phénomènes physiques

5.2. Modélisation
5.3. Limites de validité et développements complémentaires 5.4. Validation expérimentale

6. - Influence de l'écoulement circonférentiel réel

6.1. Influence de la vitesse tangentielle d'entrée

6.2. Equations de Hirs développées par D.W. Childs

6.3. Application des méthodes employées pour les paliers

7. - Étude de la stabilité

7.1. Influence des labyrinthes sur les vitesses critiques

7.2. Tournoiement d'arbre et instabilités

7.3. Labyrinthes coniques - rainurés et multiples

8. - Conclusion 


\section{Notations}

Oxyz : repère orthonormé lié au stator

$O \quad$ : centre de la surface statorique du labyrinthe côté basse pression

$\mathrm{Oz} \quad$ : axe de la turbomachine orienté vers la haute pression

$O \quad$ : centre de la surface rotorique du labyrinthe centré sur l'arbre

$\vec{e}=O O^{\prime}$ : vecteur excentricité $e=\left|O O^{\prime}\right|$ Excentricité

$O^{\prime} x^{\prime} y^{\prime} z$ : repère orthonormé avec $O^{\prime} x^{\prime}$ porté par $\vec{e}$

$\theta_{c} \quad:$ angle entre $O x^{\prime}$ et $O x$

$h \quad$ : jeu radial

$h_{o} \quad$ : jeu radial moyen pour $e=0$

$\varepsilon=e / h_{o}$ : excentricité relative $(0 \leqslant \varepsilon \leqslant 1)$

$D, R \quad$ : diamètre et rayon de la surface rotorique

$D_{S} \quad$ : diamètre de la surface statorique

$L \quad$ : longueur du labyrinthe

p : pression

$V_{a} \quad:$ vitesse axiale moyenne le long d'une génératrice
$V_{a o} \quad:$ vitesse axiale moyenne dans tout le labyrinthe pour $\varepsilon=0$

$\Delta p=p_{1}-p_{2}$ : différence de pression entre entrée et sortie du labyrinthe

$\xi \quad:$ coefficient de perte de charge à l'entrée

$\lambda \quad$ : coefficient de perte de charge par frottement $\left(\lambda=\lambda_{0}\right.$ pour $\left.h=h_{o}\right)$

$\sigma=\lambda_{0} L / h_{0}:$ coefficient de longueur relative apparente

$L / D \quad$ : longueur relative

$F, F_{x}, F_{y}, F_{x}, F_{y}$, : effort radial sur le labyrinthe et ses composantes

$V_{\theta} \quad:$ vitesse tangentielle

$V_{\theta_{1}} \quad:$ vitesse tangentielle moyenne à l'entrée du labyrinthe

$\omega \quad$ : vitesse angulaire de rotation du rotor ( $\mathrm{rd} / \mathrm{s})$

$U \quad$ : vitesse périphérique du labyrinthe

$\Omega=d \theta_{c} / d t$ : vitesse angulaire de tournoiement de l'arbre

$K, B, G$ : raideur, amortissement, inertie

$K_{x^{\prime}}=F x^{\prime} / e:$ raideur directe

$\overline{\bar{K}}_{x^{\prime}}=F x^{\prime} / \triangle p D L E$ : raideur adimensionnelle directe

$\overline{\bar{K}}_{y^{\prime}}=F y^{\prime} / \triangle p D L \varepsilon$ : raideur adimensionnelle croisée.

\section{Introduction}

L'influence des labyrinthes (*) sur le comportement dynamique des rotors de turbomachines est ressentie depuis longtemps par les constructeurs, les expérimentateurs et les utilisateurs.

Suivant les cas, ils peuvent diminuer ou augmenter les vibrations de la machine et même parfois être responsables d'instabilité pouvant entraîner sa mise hors service.

L'étude systématique de cette influence, tant sur le plan théorique que sur machines d'essai, commencée il y a 25 ans avec LOMAKIN [1] s'est poursuivie au fur et à mesure que les turbomachines industrielles étaient de plus en plus "poussées" en puissance, en dimensions et surtout en "hauteur par étage " et aussi soumises à des fonctionnements de plus en plus éloignés des conditions nominales. Citons par exemple les pompes alimentaires de centrales, les pompes-turbines réversibles et les turbopompes de fusées.

Il faut noter que les recherches effectuées en France jusqu'à présent, dans ce domaine, restent ponctuelles et n'ont en général pas été publiées.

Dans la présente communication les auteurs se sont attachés seulement à décrire les phénomènes physiques essentiels rencontrés dans les divers types de labyrinthes en insistant sur celui constitué par deux bagues cylindriques. Ils ont cherché à mettre en évidence l'intérêt et les limites des principaux modèles théoriques publiés dans la

(*) "Labyrinthe " est ici un terme général qui désigne tout dispositif à faible jeu entre rotor et stator destiné à limiter la fuite entre compartiments à pressions différentes d'une turbomachine. Il peut imposer au fluide un trajet compliqué, justifiant le terme de "labyrinthe " au sens propre... A la limite, au contraire, il s'agit de l'espace entre deux cylindres de révolution lisses et coaxiaux à l'arrêt. littérature. Il apparaît qu'un important travail reste à faire tant sur le plan théorique qu'expérimental pour répondre aux problèmes industriels actuels.

\section{Remarque :}

De façon générale, rapportées à un repère orthonormé fixe $O x y z$ ( $O z$ suivant l'axe de la machine), les composantes des efforts radiaux peuvent s'écrire sous forme matricielle, en fonction des composantes de l'excentricité $e$ de l'arbre et de leurs dérivées par rapport au temps :

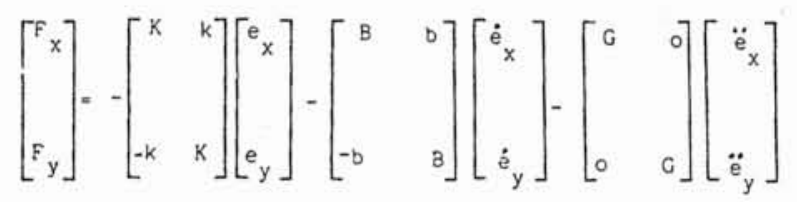

C'est à la détermination de ces coefficients de raideur, d'amortissement et d'inertie, directs et croisés et de l'influence sur eux des divers paramètres intervenant, que s'attachent les études théoriques et expérimentales.

\section{Principaux types de labyrinthes}

On rencontre dans les turbomachines hydrauliques plusieurs types de labyrinthes dont la géométrie et les conditions de fonctionnement sont très différentes. La figure 1 montre, à titre d'exemple, l'emplacement et le rôle des labyrinthes pouvant exister sur une pompe multicellulaire à hautes performances, la figure 2 représentant quelques uns des types géométriques rencontrés. 


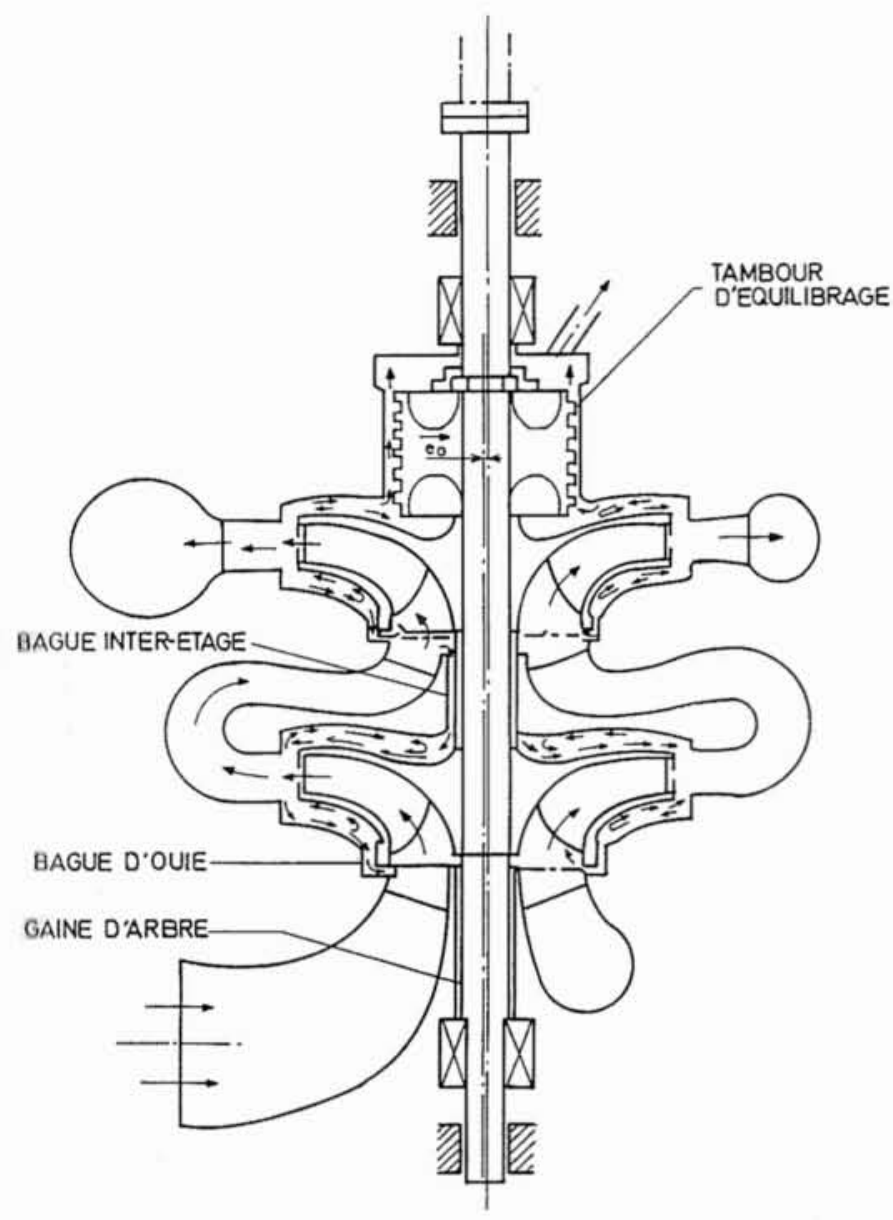

Figure 1. - Pompe multicellulaire schématique

- bague d'ouie lisse (fig. 2a) ou rainurée (fig. 2b), conique convergente (fig. 2c), en escalier (fig. $2 d$ ), ou multiple (fig. 2e);

- bague interétages soumise à une faible différence de pression quelquefois remplacées par un palier hydrodynamique ou hydrostatique;

- tambour d'équilibrage (lisse ou rainuré) destiné à compenser les poussées axiales, dans les pompes alimentaires par exemple, remplacé par un labyrinthe multiple dans d'autres cas.

\section{Raideur hydrostatique sans rotation (effet Lomakin)}

Historiquement, c'est à la suite de graves problèmes rencontrés sur des pompes alimentaires que Lomakin établit en 1957 une première modélisation des effets hydrostatiques résultant de l'écoulement dans les labyrinthes [1].

A l'époque, la raideur hydrostatique a été assimilée à une masse négative afin de conserver son caractère linéaire au calcul de vitesse critique, la force de rappel variant proportionnellement à la différence de pression aux extrémités du labyrinthe et donc au carré de la vitesse de rotation comme les forces de balourd.

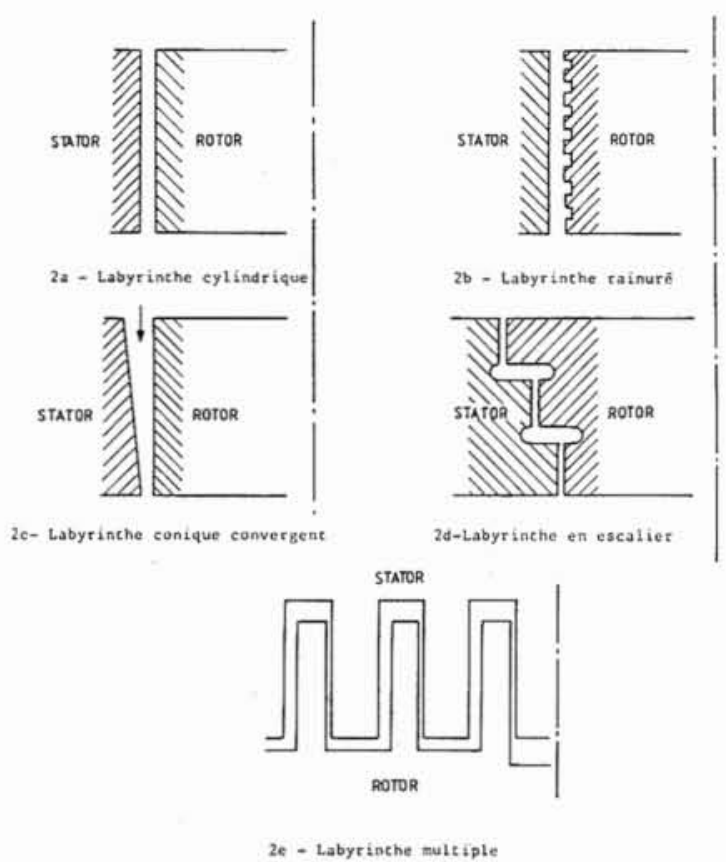

Figure 2. - Différents types de labyrinthes
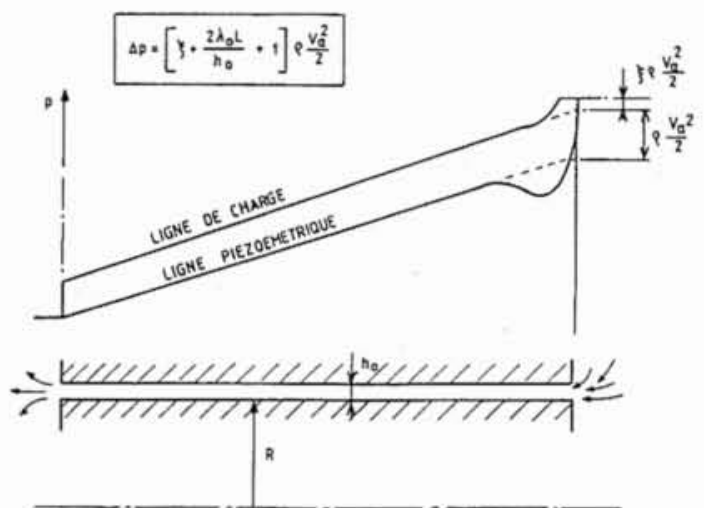

Figure 3. - Lignes piézométriques dans un labyrinthe cylindrique à jeu constant sans rotation (les vitesses dans les chambres situées aux bornes du. labyrinthe sont considérées comme négligeables).

\subsection{Ecoulement entre deux cylindres coaxiaux (jeu constant)}

L'écoulement en régime turbulent entre deux cylindres coaxiaux a fait l'objet de plusieurs études expérimentales. La figure 3 montre l'évolution de la ligne de charge et de la ligne piezométrique. Les pertes de charge se répartissent de la façon suivante: 

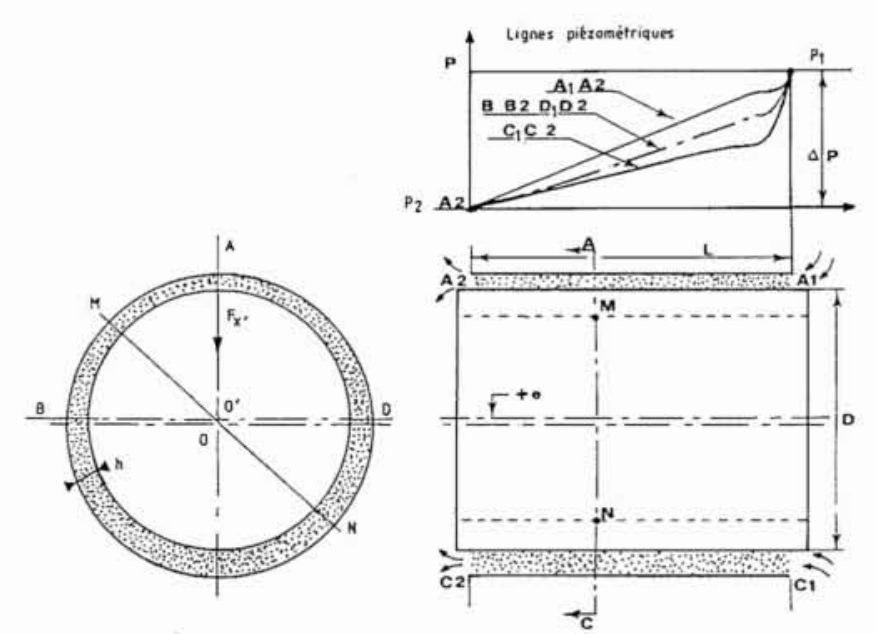

Figure 4. - Base de l'effet Lomakin (l'effet de la rotation est négligé).

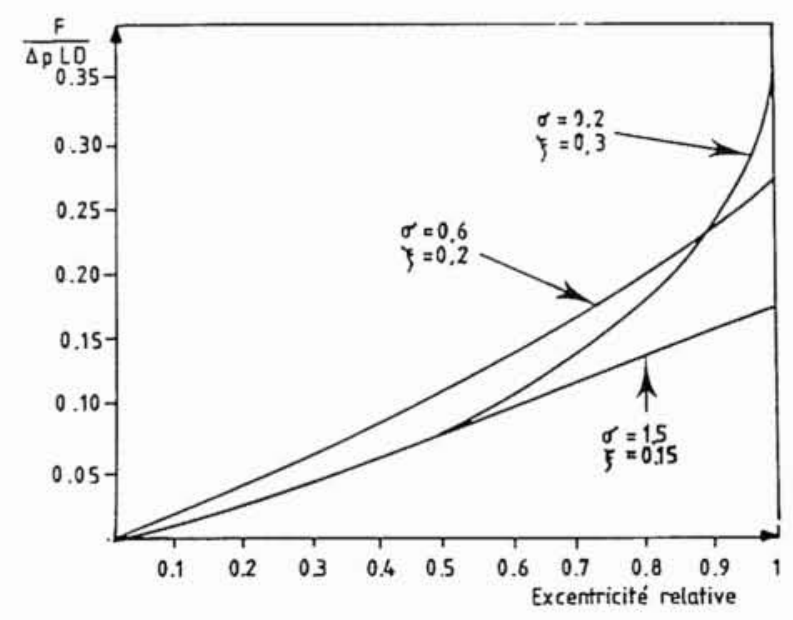

Figure 5. - Capacité de charge en fonction de l'excentricité relative $\left(\sigma=\lambda_{0} L h_{0}\right)$ calculée avec $\lambda=\lambda_{0}$ et $c=1$.

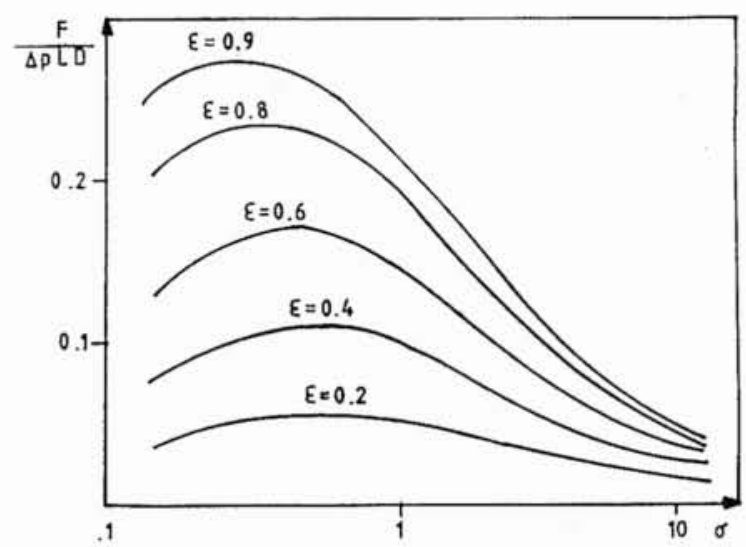

Figure 6. - Capacité de charge en fonction du coefficient de longueur relative apparent d'après la réf. [5] (le coefficient de frottement $\lambda$ varie en fonction de la vitesse axiale locale).
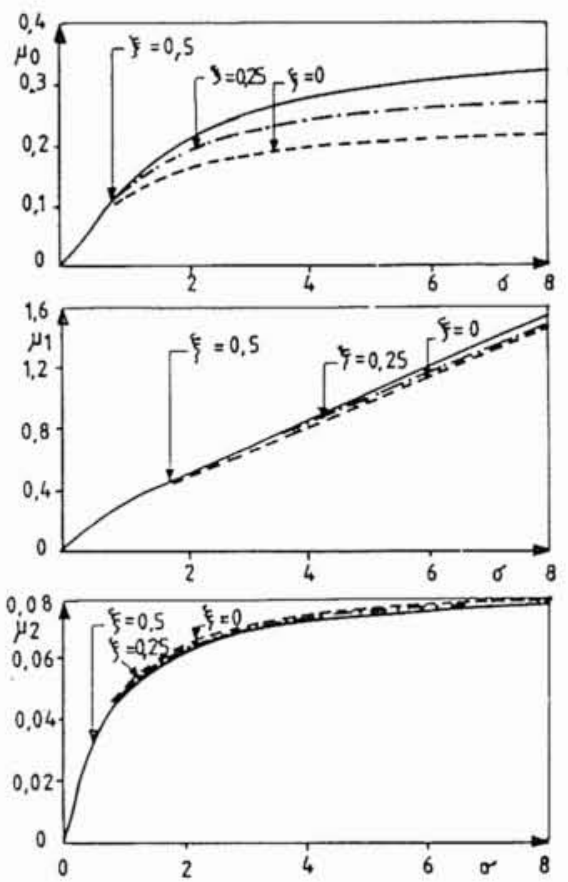

Figure 7. - Evolution des coeffients $\mu_{0}, \mu_{1}$ et $\mu_{2}$ en fonction de la longueur relative apparente $\xi$ d'après la réf. [7].
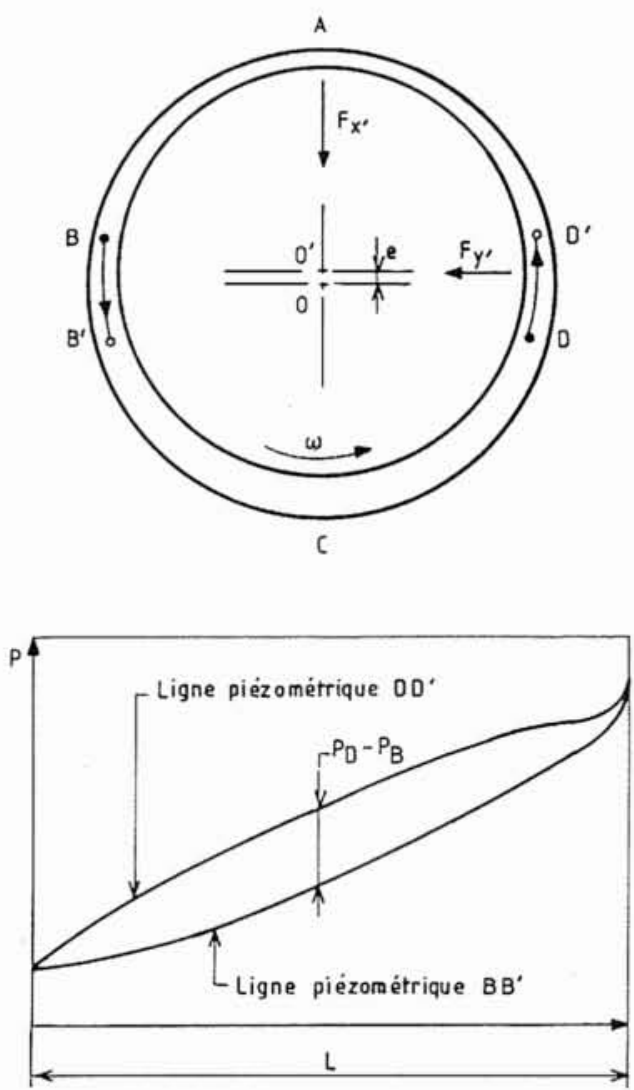

Figure 8. - Apparition d'un terme de raideur croisée. 
- perte de charge locale résultant entre autres du décollement qui peut se produire à l'entrée du labyrinthe. Dans la réalité industrielle, le coefficient de perte de charge $\xi$ est inférieur à la valeur classique de 0.5 . Cependant un écart d'estimation sur $\xi$ ne modifie pas beaucoup les résultats tant que $\sigma$ n'est pas très supérieur à 1 ;

- le coefficient $\lambda$ de perte de charge linéaire peut être calculé à partir des formules semi-empiriques proposées par Viano [2], Yamada [3] ou Hirs [4];

- la totalité de la pression dynamique est perdue en sortie de labyrinthe.

\subsection{Raideur hydrostatique (sans rotation de l'arbre)}

Le déplacement radial de la bague intérieure (liée à l'arbre) par rapport à la bague extérieure (liée au stator de la machine) provoque une dissymétrie des lignes piézométriques (fig. 4). Il en résulte pour le rotor une force de direction opposée au vecteur excentricité (raideur directe). $\mathrm{Si}$ on suppose que tous les filets fluides restent axiaux, on peut définir de façon analogue la ligne piézométrique le long de chaque génératrice en fonction du jeu radial local.

L'intégration des forces radiales élémentaires dues au champ de pression sur toute la surface cylindrique intéressée permet d'évaluer la force $F_{x}$, exercée sur la partie rotorique du labyrinthe. Il en résulte l'expression suivante pour la raideur adimensionnelle :

$$
\overline{\bar{K}}_{x^{\prime}}=\frac{F x^{\prime}}{\Delta p L D \varepsilon}=\frac{\pi \sigma(1+\xi)}{2(2 \sigma+1+\xi)^{2}-2 c(1+\xi) \varepsilon^{2}}
$$

avec $c$ légèrement inférieur à 1 et $\lambda$ constant.

La figure 5 montre que la raideur peut être considérée comme constante pour des excentricités relatives inférieures à 0.5 .

La raideur adimensionnelle $F / \Delta p D l \varepsilon$ évolue considérablement en fonction du coefficient de la longueur relative apparente du labyrinthe $\sigma=\lambda_{0} L / h_{0}$. Il apparaît que l'allongement du labyrinthe ne procure un gain de raideur significatif que pour des valeurs de $\sigma$ ne dépassant pas 0,6 à 0,7 . Au-delà la raideur adimensionnelle diminue et l'augmentation de la longueur du labyrinthe est de moins en moins "payante".

\subsection{Limites de validité et développements complémen- taires}

- Il est possible d'obtenir une expression plus précise de la raideur en prenant en compte l'influence des variations locales du nombre de Reynolds sur $\lambda$ dans le calcul du champ de pression.

- Les abaques de Viano et les formules de Yamada permettent de tenir compte de l'influence de la rotation de l'arbre sur le coefficient $\lambda$.

- Dans le cas de labyrinthes dont l'allongement relatif $L / D$ est supérieur à 0.25 , l'hypothèse de paralléllisme des filets fluides peut être mise en cause. Black propose une formule semi-empirique (voir 5.3) pour tenir compte de la réduction de raideur due à la déviation des filets fluides sous l'action des gradients de pression circonférentiels.

\section{Amortissement et inertie sans rotation}

En 1969, H.F. Black [6] propose une approche plus complète qui permet d'obtenir en plus de la raideur une expression de l'amortissement et de "l'inertie ". Il prend en compte l'effet de l'écrasement de l'écoulement dû au mouvement radial de l'arbre en supposant que l'écoulement résultant est essentiellement axial (hypothèse similaire à celle des paliers infiniment courts).

Cette approche est justifiée pour des allongements relatifs $L / D<0.2$.

Après intégration de l'équation,

$$
\frac{1}{\rho} \frac{d p}{d z}=-\frac{d V}{d t}-\frac{\lambda}{h} V^{2}
$$

( $V$ étant la valeur de $V_{a}$ modifiée par le mouvement radial).

Une linéarisation, valable pour les faibles excentricités donne des expressions analytiques pour les coefficients de raideur, d'amortissement et de masse.

$$
F=-\frac{\pi \Delta p D}{2 \lambda}\left[\mu o e+\mu_{1} \dot{e} T+\mu_{2} \ddot{e} T^{2}\right]
$$

avec $T=L / V a_{0}$ (temps de traversée du labyrinthe).

Black donne pour $\mu_{0}, \mu_{1}$ et $\mu_{2}$ les valeurs suivantes [7] :

$$
\begin{aligned}
& \mu_{0}=\frac{(1+\xi) \sigma^{2}}{(1+\xi+2 \sigma)^{2}} \\
& \mu_{1}=\frac{(1+\xi)^{2} \sigma+(1+\xi)(2.33+2 \xi) \sigma^{2}+3.33(1+\xi) \sigma^{3}+1.330^{4}}{(1+\xi+2 \sigma)^{3}} \\
& \mu_{2}=\frac{0.33(1+\xi)^{2}(2 \xi-1) 0+(1+\xi)(1+2 \xi) 0^{2}+2(1+\xi) 0^{3}+1.33 \sigma^{4}}{(1+\xi+2 \sigma)^{4}}
\end{aligned}
$$

A noter que :

$$
\mu_{0}=\frac{2 \sigma}{\pi} K_{x^{\prime}}=\frac{2 \lambda_{0} \mathrm{~L}}{\pi h o} \bar{K}_{x^{\prime}}
$$

ce qui est cohérent avec l'expression du paragraphe 3.2.

Les courbes $\mu_{0}, \mu_{1}, \mu_{2}$ en fonction de $\sigma$ sont toujours croissantes alors que $K_{x}$ passe par un maximum (fig. 7).

\section{Influence de la rotation avec écoulement de Couette}

\subsection{Phénomènes physiques}

La rotation de l'arbre induit un écoulement circonférentiel qui entraîne, lorsque le jeu radial n'est pas constant, un déséquilibre des pressions dans la direction perpendiculaire au vecteur excentricité. La figure 8 schématise la force $F_{y}$. dite croisée, qui en résulte. Cette force tend à faire tourner le point $O^{\prime}$ autour du point $O$ dans le sens de la rotation $\omega$ de l'arbre. Elle peut s'exprimer à l'aide d'un coefficient de raideur croisée

$$
\overline{\bar{K}}_{y^{\prime}}=\frac{F y^{\prime}}{\Delta p D l \varepsilon}
$$

\subsection{Modélisation}

En supposant que l'écoulement circonférentiel moyen a une vitesse angulaire égale à la moitié de celle $\omega$ du rotor, il est possible d'appliquer la théorie exposée au chapitre précédent dans un repère tournant à la vitesse angulaire $\omega / 2$ (voir plus loin en 6 , l'examen de cette hypothèse). 


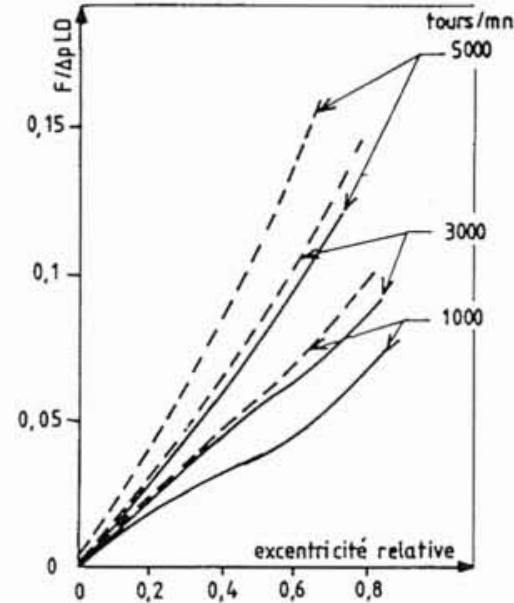

Figure 9. - Comparaison des capacités de charges théoriques et expérimentales obtenues par H.F. Black [7] $(\Delta p \simeq 3,5 \mathrm{~b} ; L=50,8 \mathrm{~mm}$; $\left.D=50,8 \mathrm{~mm} ; h_{0}=0,27 \mathrm{~mm}\right)$.

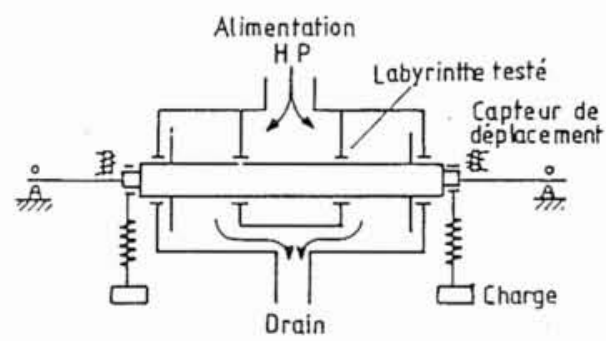

Figure 10. - Schéma de principe de la machine d'essai de H.F. Black. Plusieurs autres machines ont été construites [8], [9], [10], [12], [13], [14] ...
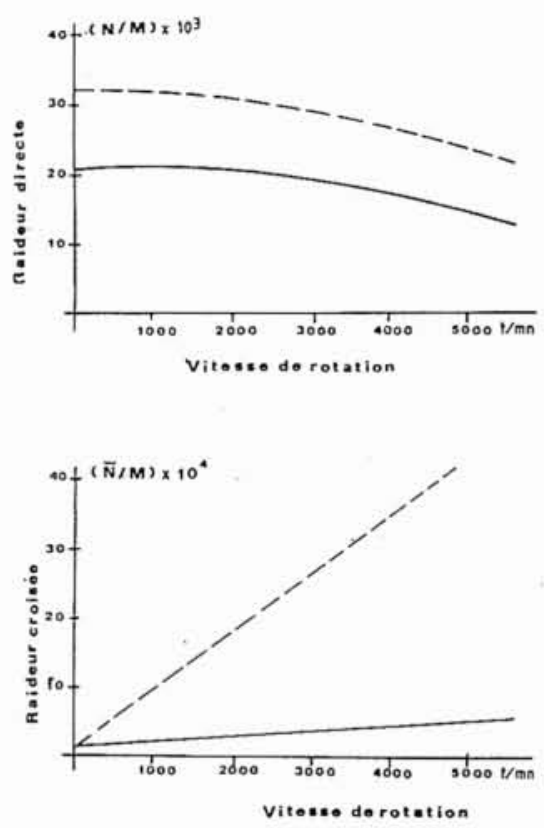

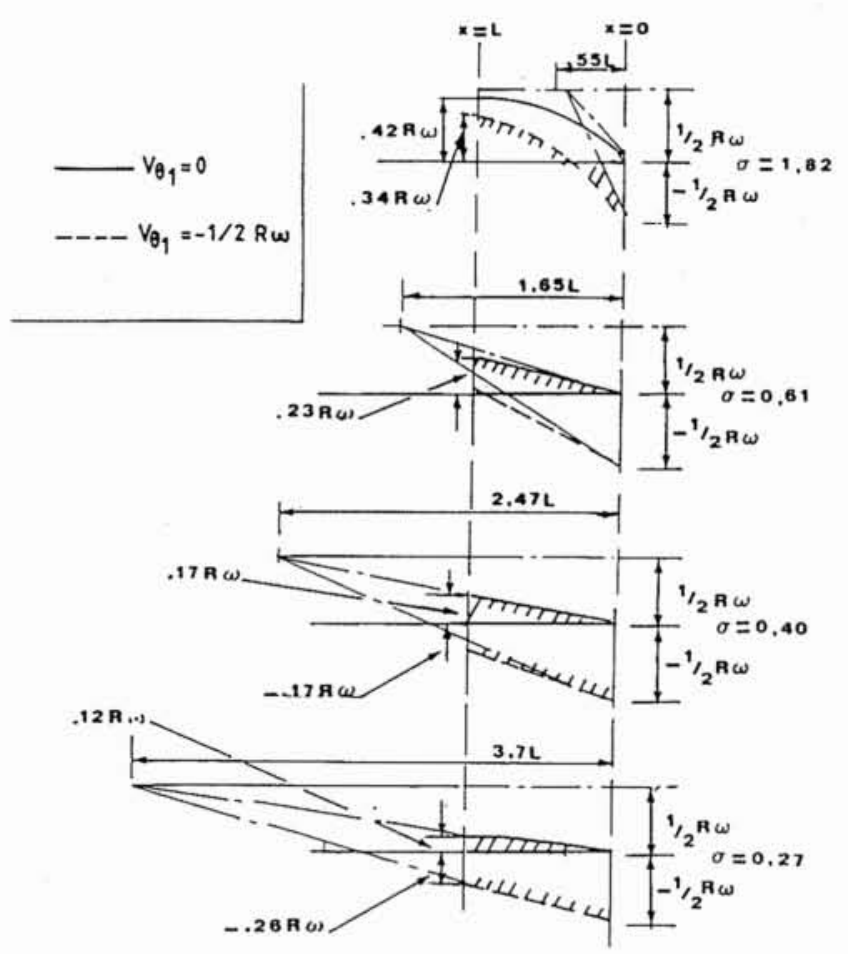

Figure 12. - Evolution de la vitesse circonférentielle moyenne en fonction de sa valeur à l'entrée du labyrinthe d'après Black [11].
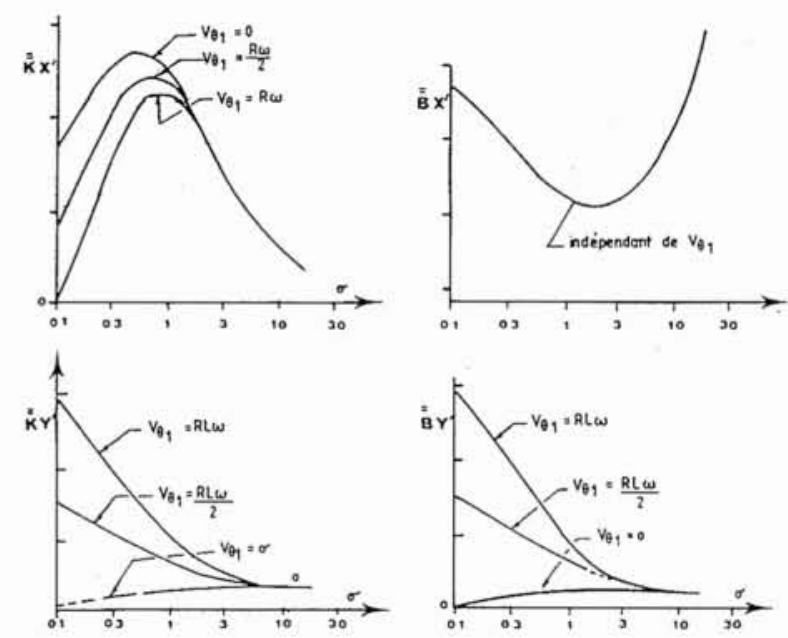

Figure 13. - Influence de la vitesse circonférentielle à l'entrée du labyrinthe sur les coefficients dynamiques d'après D.P. Fleming [12] $\left(L / D=0.5 ; R \omega / V_{\infty}=0.5\right)$

Figure 11. - Coefficients dynamiques obtenus expérimentalement par $\mathrm{R}$. Nordmann et $\mathrm{H}$. Massmann [8]. Les valeurs théoriques en pointillés ont été calculées par les formules de Black. Les courbes expérimentales en traits pleins sont des courbes moyennes. Les caractéristiques de la machine sont : $L=35$ $\mathrm{mm} ; D=42 \mathrm{~mm} ; h_{0}=0.35 \mathrm{~mm} ; \Delta p=2,23 \mathrm{~b}$ 
En ramenant les valeurs précédentes dans le repère $O x y z$, Black a ainsi obtenu une expression des termes directs et croisés qui tient compte de la rotation du fluide.

Elle peut s'écrire sous forme matricielle comme suit :

$$
\left[\begin{array}{l}
F \\
x^{\prime} \\
F y^{\prime}
\end{array}\right]=-\frac{\pi D P}{2 \lambda}\left(\left[\begin{array}{ll}
\mu_{0}-\frac{1}{4} \mu_{2} \omega^{2} T^{2} & \frac{1}{2} \mu_{1} \omega_{1} T \\
-\frac{1}{2} \mu_{1} \omega T & \mu_{0}-\frac{1}{4} \mu_{2} \omega^{2} T^{2}
\end{array}\right]\left[\begin{array}{l}
e_{x} \\
e_{y}
\end{array}\right]\right.
$$

$$
\left.+\left[\begin{array}{cc}
\mu_{1} T & \mu_{2} \omega T^{2} \\
-H_{2} \omega T^{2} & \mu_{1} T
\end{array}\right]\left[\begin{array}{l}
\dot{e}_{x} \\
\dot{e}_{y}
\end{array}\right]+\left[\begin{array}{cc}
\mu_{2} T^{2} & 0 \\
0 & H_{2} T^{2}
\end{array}\right]\left[\begin{array}{c}
\ddot{e}_{x} \\
\ddot{e}_{y}
\end{array}\right]\right)
$$

Ces matrices peuvent être introduites dans un code de calcul du comportement dynamique d'une ligne d'arbre complète.

\subsection{Limites de validité et développements complémen- taires}

Dans les labyrinthes réels qui sont loin d'être « infiniment courts ", les gradients de pression circonférentiels induisent un écoulement circonférentiel d'autant plus important que l'allongement relatif est important.

Black et Jensen [7] ont effectué des calculs plus élaborés qui prennent en compte un écoulement circonférentiel faible devant l'écoulement axial. Ils en ont déduit des formules de correction des coefficients $\mu_{0}, \mu_{l}, \mu_{2}$.

$$
\begin{aligned}
& \mu_{0}\left(\frac{L}{R}\right)=\frac{\mu_{0}}{1+0.28(L / R)^{2}} \\
& \mu_{0}\left(\frac{L}{R}\right)=\frac{\mu_{1}}{1+0.23(L / R)^{2}} \\
& \mu_{2}\left(\frac{L}{R}\right)=\frac{\mu_{2}}{1+0.06(L / R)^{2}}
\end{aligned}
$$

La méthode employée qui repose sur l'intégration numérique des équations non-linéaires dans le cas d'une excentricité fixe donne l'évolution des coefficients dynamiques pour des valeurs de l'excentricité relative allant jusqu'à 0.8 .

\subsection{Validation expérimentale}

La dispersion des résultats expérimentaux publiés montre qu'il est difficile d'obtenir des valeurs fiables pour les coefficients dynamiques. Les raisons principales sont les suivantes :

- machines d'essais trop petites, non représentatives;

- mauvais contrôle des paramètres (notamment la vitesse tangentielle d'entrée du fluide dans le labyrinthe);

- instrumentation inadaptée, insuffisante ou difficile à maitriser (en particulier: capteurs de déplacement et capteurs de pression dans des petits interstices).

A titre d'exemple, nous avons reproduit le schéma de la machine d'essais de H.F. Black (fig. 10). Nous avons relevé des résultats de Black (fig. 9) et ceux plus récents de Nordmann. Ces résultats (fig. 11) montrent qu'en général, les calculs effectués par les méthodes dérivées de celles de Black fournissent des valeurs un peu excessives pour les raideurs. Toutefois l'évolution des coefficients en fonction de la vitesse de rotation est conforme à la théorie. Dans une communication publiée en 1982 [9], Gopalakrishnan montre qu'il a obtenu une très bonne prévision des vitesses critiques d'une machine d'essai de taille beaucoup plus importante que les machines de laboratoire de Black et Nordmann.

En ce qui concerne l'amortissement, les résultats théoriques semblent concorder de façon suffisamment satisfaisante avec les résultats expérimentaux. Il faut noter que les résultats expérimentaux obtenus récemment par Childs [10] montrent la même tendance.

\section{Influence de l'écoulement circonférentiel réel}

\subsection{Influence de la vitesse tangentielle d'entrée}

Depuis 5 ans, les auteurs ont cherché à améliorer la modélisation des écoulements circonférentiels.

D'une part l'augmentation des vitesses de rotation conduit dans bien des cas à des nombres de Reynolds circonférentiels du même ordre de grandeur que les nombres de Reynolds axiaux, ce qui montre l'importance des termes de convection circonférentiels.

D'autre part, il a été montré théoriquement et expérimentalement [11] qu'il faut en général une distance assez importante pour que l'écoulement circonférentiel moyen atteigne la vitesse circonférentielle $V_{\theta}$ asymptotique $R \omega / 2$, si $V \theta_{1}$ à l'entrée en diffère beaucoup (fig. 12). Il en résulte une forte influence de la vitesse circonérentielle d'entrée sur certains coefficients dynamiques en particulier sur la raideur croisée (cf. fig. 13 publiée par Fleming [12]).

\subsection{Equations de Hirs développées par D.W. Childs}

En 1973, Hirs [4] a établi des expressions semi-empiriques donnant les coefficients de frottement locaux pour les écoulements turbulents axiaux et circonférentiels pour les films minces.

L'introduction de ces coefficients dans les équations de quantité de mouvement conduit aux équations dites de Hirs :

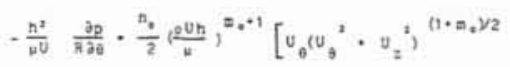

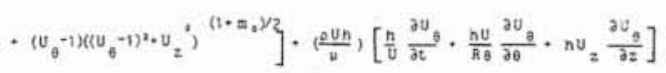

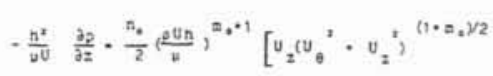

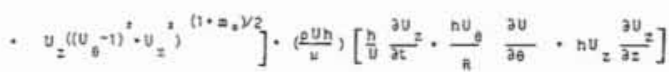

avec $n_{0}$ et $m_{0}$ constantes semi empiriques et $U_{\theta}=V_{\theta} / U$; $U_{i}=V_{i} / U$

Nota: Pour les labyrinthes d'ouie (fig. 1), l'hypothèse $V_{\text {ต }}=R \omega / 2$ semble justifiée, par contre pour les bagues interétage on a $V_{\theta \mathrm{l}}=0$, ce qui réduit considérablement le coefficient de raideur croisée. 
D.W. Childs a appliqué ces équations aux labyrinthes et a développé une méthode de résolution valable pour les faibles excentricités qui prend en compte l'évolution axiale et azimuthale de la vitesse circonférentielle [10].

Il a pu ainsi obtenir des valeurs plus satisfaisantes des coefficients croisés pour des valeurs de la vitesse circonférentielle d'entrée différentes de la valeur asymptotique $R \omega / 2$. Il semble cependant que dans le cas où le nombre de Reynolds circonférentiel est très élevé ou bien s'il se produit des variations rapides de la vitesse circonférentielle moyenne la théorie de Hirs soit insuffisante pour décrire l'écoulement turbulent dans le labyrinthe.

\subsection{Application des méthodes employées pour les paliers}

Certains auteurs (Kaneko [13], Falco [14]) ont tenté d'employer des codes de calcul développés pour les paliers fonctionnant en régime turbulent qui résolvent les équations non linéaires de Constantinescu (équations de Reynolds avec des coefficients de frottement turbulents semi empiriques). Cette méthode ne prend pas en compte les termes d'inertie. Comme le montre les comparaisons calcul-expérience de Kaneko, cette méthode ne se justifie que pour des labyrinthes à jeux très faibles. Cependant, il est probable qu'elle puisse fournir des résultats intéressants à très forte excentricité où ses conditions de validité sont mieux respectées et où une résolution non linéaire s'impose.

\section{Etude de la stabilité}

\subsection{Influence des labyrinthes sur les vitesses critiques}

L'influence la plus connue des labyrinthes est la modification des vitesses critiques due aux termes de raideur directe. Dans un certain nombre de cas, cet effet a même été recherché pour relever les vitesses critiques des lignes d'arbre.

Si ceci est valable pour les turbopompes de fusées, où l'on doit rechercher à tout prix le minimum de poids et par suite, la vitesse de rotation maximale, mais pour une courte durée d'utilisation; cela l'est beaucoup moins pour des machines industrielles où l'on doit tenir compte de l'usure normale pendant des milliers d'heures de fonctionnement par l'eau utilisée.

\subsection{Tournoiement d'arbre et instabilités}

La seconde influence importante des labyrinthes sur le comportement des rotors de turbomachines est la création ou la modification des forces croisées (efforts tangentiels s'appliquant sur l'arbre excentré perpendiculairement à l'excentricité $e$ ). Ces forces viennent s'ajouter aux diverses forces déphasées (d'origines hydrauliques ou engendrées dans les paliers) pour favoriser un tournoiement en général subsynchrone de l'arbre. (Le vecteur $e$ tournant à la vitesse angulaire $\Omega=d \theta_{f} / d t$ par rapport au stator). Les coefficients établis par Black [6] permettent de mettre en évidence que les labyrinthes peuvent être responsables de phénomènes de fouettement analogues à ceux rencontrés dans les paliers.

La figure 14 montre que lorsque la vitesse de rotation de l'arbre est un peu supérieure au double d'une vitesse critique, cette vitesse critique peut être excitée.

\subsection{Labyrinthes coniques - rainurés et multiples}

Labyrinthes coniques: Dans de nombreux cas industriels, les efforts d'origine hydraulique ou mécanique entrainent un fléchissement relativement important de l'arbre. Les axes des parties rotoriques et statoriques des labyrinthes ne peuvent plus alors être considérés comme parallèles.

La figure 15 montre que dans une telle configuration il peut apparaître au-delà d'une certaine excentricité une raideur hydrostatique négative source d'instabilités.

Un labyrinthe conique convergent permet de réduire les risques d'apparition de ce phénomène. Les études théoriques et expérimentales effectuées à la NASA (Fleming) montrent que ce type de labyrinthe procure une raideur plus importante qu'un labyrinthe cylindrique mais que l'amortissement est réduit et que le débit de fuite est accru.

Pour les mêmes raisons, il peut être souhaitable de prévoir des jeux décroissants dans le cas de longs labyrinthes en escalier.

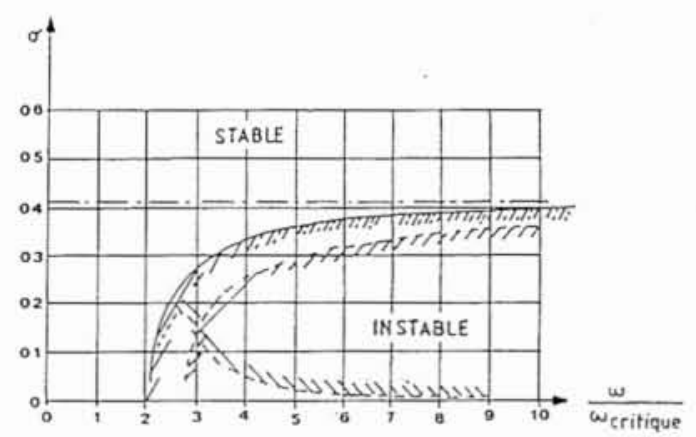

Figure 14. - Limites de stabilité dans le cas d'une roue placée entre deux paliers rigides d'après H.F. Black [8]

Limite de stabilité sans aucune source d'amortissement en dehors des labyrinthes.

Limite de stabilité avec un faible amortissement extérieur aux labyrinthes.

En pratique, il faut aussi prendre en compte les autres forces d'excitation hydrauliques.
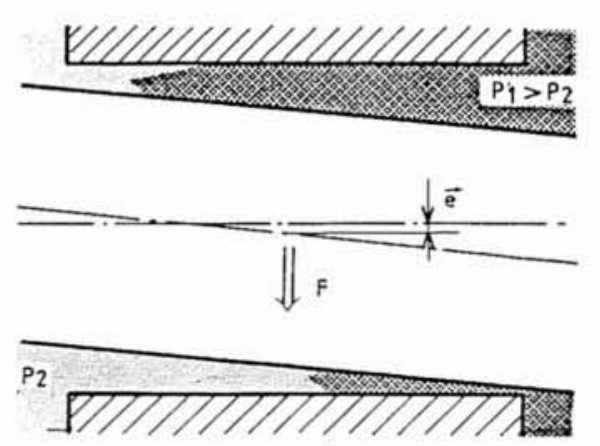

Figure 15. - Apparition d'une raideur négative source d'instabilité dans le cas de non parallélisme des bagues statorique et rotorique. 
Labyrinthes rainurés: Dans certains cas industriels on est amené à rainurer la partie statorique ou rotorique afin de réduire l'influence des labyrinthes sur le comportement dynamique de la ligne d'arbre. Si la forme des rainures est optimisée, il est possible d'obtenir un débit de fuite égal et même plus faible qu'avec un labyrinthe lisse. Les rainures réduisent considerablement les gradients de pression circonférentiels, il en résulte une raideur sensiblement plus faible.

Par ailleurs, les risques d'instabilités dus au désalignement évoqués au chapitre précédent sont aussi réduits. Cependant, de façon analogue à ce qui se produit sur les labyrinthes à lèvres des turbines à vapeur et des compresseurs, il peut apparaître des forces croisées assez importantes.

Labyrinthes multiples: Sur les turbomachines hydrauliques de grande dimension fonctionnant sous forte hauteur, il est souvent indispensable d'adopter des labyrinthes multiples, notamment pour réduire la poussée axiale.

Le dimensionnement de ce type de labyrinthe nécessite quelques précautions. En effet, certaines configurations peuvent être instables (Sédille, Priesniz [16]), c'est essentiellement pour cette raison qu'ils ont généralement disparu sur les pompes alimentaires.

Il est de toute façon souhaitable de dimensionner largement les chambres existant entre chaque étage de laminage afin de réduire les gradients de pression circonférentiels. Il faut aussi répartir convenablement les jeux.

\section{Conclusion}

La présente étude, forcément très limitée, a cherché avant tout à montrer que des recherches importantes ont déjà été effectuées dans le monde entier sur ce problème parfois crucial de l'influence des labyrinthes sur le fonctionnement des turbomachines et à sensibiliser encore plus les ingénieurs français sur cette question.

Les constructeurs ont commencé, depuis plusieurs années, à tenir compte, de certains aspects de l'influence des labyrinthes sur le comportement dynamique des rotors des machines les plus "poussées", notamment en ce qui concerne l'influence de la raideur directe sur les vitesses critiques.

Cependant, de nombreux développements complémentaires restent à effectuer pour résoudre les problèmes industriels actuels parmi lesquels nous pouvons citer, à titre d'exemple :

- la prévision du comportement dynamique des grandes machines hydrauliques qui nécessite une modélisation complète du comportement des labyrinthes valable même pour les fortes excentricités;

- la conception des nouvelles turbopompes de fusées qui requiert une bonne prévision des termes croisés à très haute vitesse de rotation afin de maitriser les phénomènes d'instabilités.

Sur le plan théorique, il sera vraisemblablement nécessaire de prendre en compte des paramètres géométriques tels que faux-rond, décentrement, désaxemnent, défaut de parallélisme, différences de rugosité, des bagues rotor et stator et d'envisager une représentation plus précise de l'écoulement turbulent dans les labyrinthes.

Sur le plan expérimental il sera indispensable d'éliminer les machines d'essais de trop petite taille, de contrôler de façon précise la vitesse circonférentielle d'entrée dans le labyrinthe et de tenter d'analyser l'influence des paramètres géométriques mentionnés ci-dessus.

Des progrès significatifs sur ces points nécessiteront certainement l'union des efforts de tous, constructeurs et organismes concernés.

\section{Bibliographie}

[1] A.A. LOMAKIN. - Determination of the critical speed of a pump impeller with allowance for the forces arising in the seals. (Collected papers, Leningrad Metal works, Steam and Gas Turbine Engineering) $N^{\circ} 5,1957$.

[2] VIANO. - Pertes dans les labyrinthes cylindriques lisses. $L a$ Houille Blanche, $\mathrm{N}^{\circ} 1 / 1970$.

[3] Y. Yamada, K. Nakabayashi, K. Maeda. - Pressure drop measurements of the flow through Exentric Cylinders with rotating inner Cylinders, Bulletin of JSME, Vol. 12, $\mathrm{N}^{\circ} 53$, 1969.

[4] G.G. Hirs. - A "bulk" flow theory for turbulence in lubricant films. Trans. ASME, serie $F$, Vol. 95, $\mathrm{N}^{\circ} 2$, April 1973.

[5] P.E. Allaire, C.C. Lee, E.J. Gunter. - Dynamic of short excentric plain seals with high axial Reynolds number. $J$. Spacecraft and rockets, Vol. 15, N $6,1978$.

[6] H.F. BLACK. - Effects of hydraulic forces in annular pressure seals on the vibrations of centrifugal pump rotors. Journ. of mech. Eng. Sciences, 1969, Vol. 11, N².

[7] H.F. BLACK, D.N. JENSEN. - Dynamic hydrid bearing characteristics of annular controlled leakage seals. Proc. Inst. Mech. Eng., Vol. 184, Pt 3N, 1969-70.

[8] R. NORDMANN, H. MASSMANN. - Identification of stiffness, damping and mass coefficients for annular seals. Inst. Mec. Eng. Conference, York, Sept. 1984.

[9] S. Gopalakrishnan, R. Fehl.an, J. Lorett, - Critical speed in centrifugal pumps. ASME Paper 82-GT-277.

[10] D.W. CHILDS. - Finite length solutions for the rotor dynamic coefficients of contant clearance and convergent tapered annular seals. Inst. Mec. Eng. Conf., York, Sept. 1984.

[11] H.F. Black, P.E. Allaire, L.E. BARRETT. - Inlet flow swirl in short turbulent annular seal dynamics. 9th Conf. on fluid Sealing, Noordwijkerhout, Netherlands, April 1981.

[12] D.P. Fleming. - Effect of seal on rotor systems. Shock and vibration Bulletin, Vol. 52, Part 1, May 1982.

113] S. KaneKo, Y. Hor1, M. TANAKA. - Static and dynamic characteristics of annular plain seals. Inst. of Mec. Eng. Conf., York, Sept. 1984.

[14] M. Falco, S. Mimmi, B. Pizzigoni, G. Marenco, G. Negri. - Plain seal dynamic behaviour - experimental and analytical results. Inst of Mec. Eng. Conf.. York, Sept. 1984.

[15] M.L. Adams, E. MaKay, I.A. Diaz-Tous. - Measurement of insterstage fluid annular dynamical properties. Rolor dynamic instability problems in high performance turbomachinery; Texas Univ., May 1982.

[16] Chr. Priesnitz. - L'influence des bagues d'étanchéité simples et triples sur la position des vitesses critiques de rotation et sur le comportement en rotation des rotors de pompe. Pumpen und Verdichter, RDA Halle, 1967. 


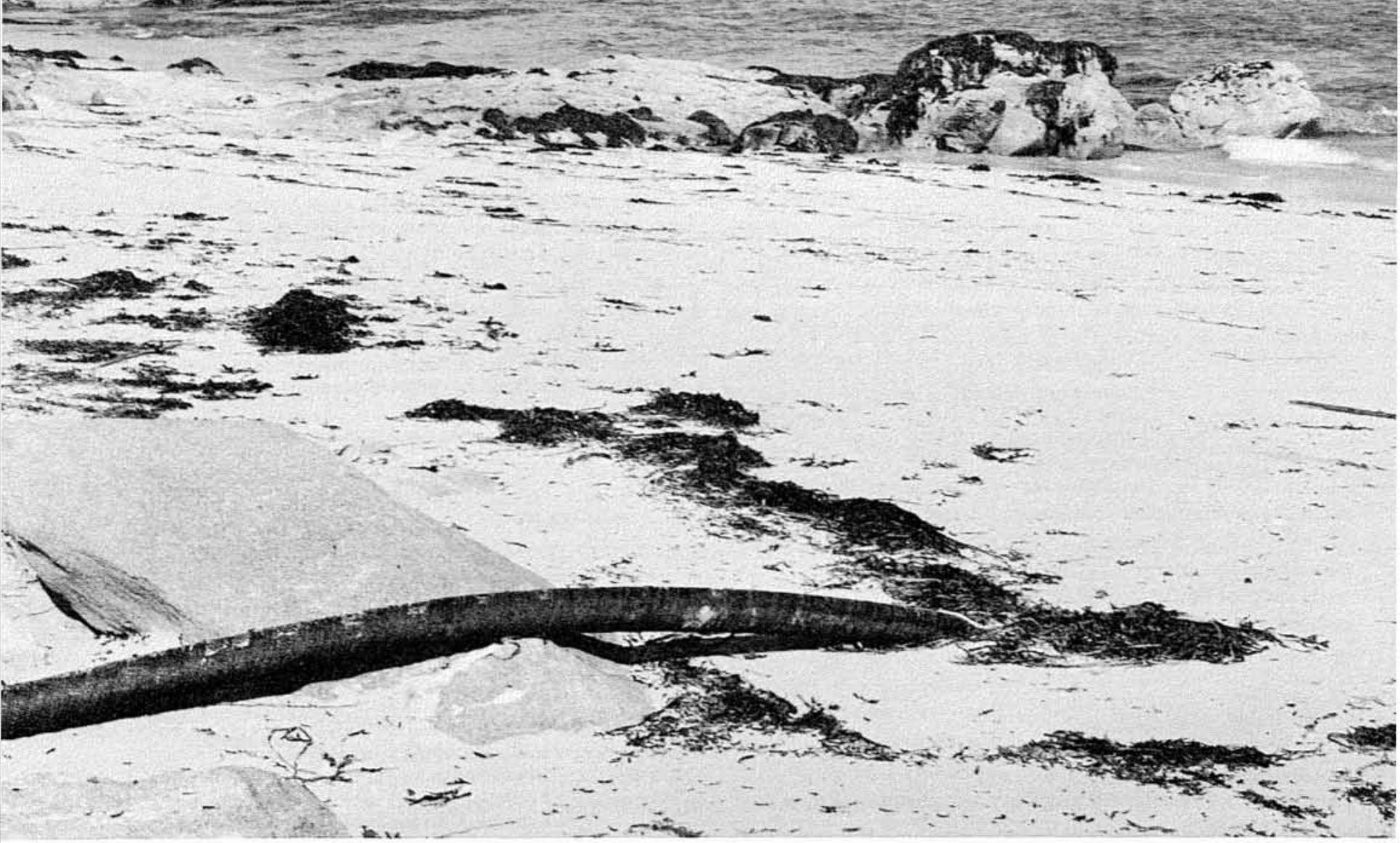

